\title{
Chaotic Spectra of Classically Integrable Systems
}

\author{
P. Crehan \\ Dept. of Mathematical Physics, University College Dublin, \\ Belfield, Dublin 2, Ireland \\ PCREH89@OLLAMH.UCD.IE \\ Dept. of Mathematics, Faculty of Science, University of Kyoto, \\ Kyoto 606, Japan
}

\begin{abstract}
We prove that any spectral sequence obeying a certain growth law is the quantum spectrum of an equivalence class of classically integrable non-linear oscillators. This implies that exceptions to the Berry- Tabor rule for the distribution of quantum energy gaps of classically integrable systems, are far more numerous than previously believed. In particular we show that for each finite dimension $k$, there are an infinite number of classically integrable $k$-dimensional non-linear oscillators whose quantum spectrum reproduces the imaginary part of zeros on the critical line of the Riemann zeta function.
\end{abstract}

PCACS: Numbers 05.45, 03.65, 02.30.Dk, 02.30.Gp, 02.10.Lh

\section{Introduction}

An important theme in the quantum theory of classically chaotic systems is the relationship between the qualitative behaviour of the classical system and statistical properties of its quantum mechanical spectrum [1]. A much studied statistic in this regard is the distribution of energy level spacings. According to a result of Berry and Tabor the values of the energy gaps of a generic integrable system are Poisson distributed [2]. In general this differs remarkably from the statistical distribution of energy gaps in the case of classically chaotic systems. These have been studied numerically and can be described by Wigner, GOE or GUE rather than Poisson statistics [1]. 
An exception to the result of Berry and Tabor occurs in the case of the harmonic oscillator. However Razavy investigated a family of integrable perturbations of the harmonic oscillator and found that this departure from Poisson statistics is non-generic in the sense that perturbing away from the harmonic oscillator, the energy gaps quickly become Poisson distributed [3] . Another exception to the Berry-Tabor result was pointed out by Casati, Chirikov and Guarneri, and occurs for the case of a free particle in a rectangular well [4]. Nevertheless Seligman and Verbaarshot observe that for potential wells close to the rectangular well, the distribution of energy gaps is close to Poisson. They conclude that in this case also departure from Poisson statistics is non-generic [5]. The general perception is that despite exceptional cases such as the harmonic oscillator and the free particle in a rectangular well, the statistics of quantum energy gaps of classically integrable systems are universally described by the Poisson distribution.

In this paper we demonstrate that given a spectral sequence obeying a certain growth law, there exists an infinite family of classically integrable Hamiltonians whose quantum spectrum coincides with this sequence. This shows that a wide range of exceptions to the Berry-Tabor rule are possible and that any quantum system whose spectrum obeys such a growth law, can be simulated by a family of classically integrable non-linear oscillators. In particular we derive a result concerning the hypothesis of Berry about the qualitative behaviour of an unknown classical system whose quantum energy levels are given by the imaginary part of the zeros on the critical line of the Riemann-zeta function [6]. Berry argues that this classical system should be chaotic. We prove that this unknown classical system is not unique and need not be chaotic. In fact we show that an infinite number of classically integrable non-linear oscillators are capable of reproducing these zeros when quantised.

\section{One dimensional case}

To justify our claims we require a number of theorems based on the following lemma and its generalisation.

LEMMA 1 Given a sequence of complex numbers $\left\{\zeta_{n}: 0 \leq n \in Z\right\}$ obeying a growth law $\left|\zeta_{n}\right|<\exp (a+b n)$ for some $a \in \Re, b \in \Re^{+}$, there exists an 
equivalence class $S$ of entire functions of the complex plane such that for each $s \in S$ we have $s(n)=\zeta_{n}$.

We prove this by considering

$$
s(z)=\sum_{n=0}^{\infty} \zeta_{n} f_{n}(z)
$$

where for $0 \leq n \in Z, 0<\epsilon \in \Re$ and $z \neq n$,

$$
f_{n}(z)=\exp ((z-n)(2 \pi+b+\epsilon)) \frac{\sin (2 \pi(z-n))}{2 \pi(z-n)} .
$$

Strictly speaking $f_{n}$ has a singularity at $z=n$. The singularity is however removable and so defining $f_{n}(n)=1, f_{n}$ becomes an entire function of the complex plane. Eventually we will show that $s$ is well defined by the series in eqn(1), and that it too is an entire function of the complex plane. Observing that $f_{n}(m)=\delta_{n m}$ for $0 \leq n, m \in Z$, the property $s(n)=\zeta_{n}$ follows from the definition of $f_{n}$ and an explicit evaluation of the sum at $z=n$. This construction provides a single repesentative member of the equivalence class $S$. The difference between any two representatives is an entire function of the complex plane which vanishes on the non-negative integers. This set is infinite and denoting it by $S_{0}$, the equivalence class is given by $S=s+S_{0}$. All that now remains is to show that $s$ is entire.

The domain $\{z \in C:|z|<\rho \in \Re\}$ will be denoted $D_{\rho}$. Using $A\left(D_{\rho}\right)$ to represent the analytic functions on $D_{\rho}$, the entire functions are naturally denoted $A(C)$. The Weierstrass M-test provides a criterion for when the infinite sum of functions which are analytic on some domain $D$, converges to a function which is analytic on $D$. Specifically if $\left\{g_{n}\right\}$ is a sequence in $A\left(D_{\rho}\right)$, and if there exists a sequence of positive real numbers $\left\{A_{n}\right\}$ with the property $A_{n} \geq\left\|g_{n}\right\|=\sup _{z \in D_{\rho}}\left|g_{n}(z)\right|$, such that $\sum_{n=0}^{\infty} A_{n}<\infty$, then $\sum_{n=0}^{\infty} g_{n}$ converges to $g \in A\left(D_{\rho}\right)$. To prove that $s$ is analytic on an arbitrary $D_{\rho}$, we apply this test to the sequence defining $s$ in eqn(1). Each term lies in $A\left(D_{\rho}\right)$ since each is a constant multiple of an entire function. Using the fact that $|\sin (z) / z| \leq \exp (|z|)$ we have for $z \in D_{\rho}$

$$
\left|\zeta_{n} f_{n}(z)\right| \leq\left|\zeta_{n}\right||\exp ((z-n)(2 \pi+b+\epsilon))|\left|\frac{\sin (2 \pi(z-n))}{2 \pi(z-n)}\right|
$$




$$
\begin{aligned}
& \leq \exp (a+b n) \exp ((|z|-n)(2 \pi+b+\epsilon)) \exp (2 \pi|z-n|) \\
& \leq \exp (a+b n) \exp ((|z|-n)(b+2 \pi+\epsilon)) \exp (2 \pi(|z|+n)) \\
& =\exp (a+|z|(4 \pi+b+\epsilon)) \exp (-\epsilon n) \\
& \leq \exp (a+\rho(4 \pi+b+\epsilon)) \exp (-\epsilon n) .
\end{aligned}
$$

To apply the Weierstrass M-test we take $A_{n}=c_{\rho} \exp (-\epsilon n)$ where the constant $c_{\rho}=\exp \left(a+\rho(4 \pi+b+\epsilon)\right.$. The sum $\sum_{n=0}^{\infty} A_{n}$ converges and so $s \in A\left(D_{\rho}\right)$. Since $s \in A\left(D_{\rho}\right)$ for any $\rho \in \Re^{+}$we have $s \in A(C)$ and lemma 1 is proven.

Although there is a different $s$ for each valid choice of $a, b$ and $\epsilon$, the corresponding equivalence class $S$ is independent of these values. Lemma 1 leads almost directly to the following theorem.

THEOREM 1 Given a real sequence $\left\{E_{n}: 0 \leq n \in Z\right\}$ which obeys a growth law $\left|E_{n}\right| \leq \exp (a+b n)$ for some $a, b \in \Re^{+}$, there exists an equivalence class of classically integrable non- linear oscillators $H_{C}$ such that if $h^{0}$ is the Hamiltonian of the simple harmonic oscillator, each $h_{C} \in H_{C}$ is of the form $h_{C}\left(h^{0}\right)$ and its quantum spectral sequence is given by $E_{n}$.

To see this construct $s$ as in lemma 1 so that $s(n)=E_{n}$ for $0<n \in Z$. The equivalence class of classical Hamiltonians $H_{C}$ comes from replacing $z$ with $h^{0}=\left(q^{2}+p^{2}\right) / 2$ in each representative $s \in S$. As $h^{0}$ is the Hamiltonian of the simple harmonic oscillator, each $h_{C} \in H_{C}$ is the Hamiltonian of a classically integrable non-linear oscillator.

Since $s \in A(C)$, each $h_{C} \in H_{C}$ can be identified with its Taylor expansion in $h^{0}$ which is everywhere convergent. This allows us to write $h_{C}=\sum_{0}^{\infty} c_{i}\left(h^{0}\right)^{i}$ where each $c_{i} \in \Re$, and to define a corresponding quantum Hamiltonian operator

$$
H_{Q}=\sum_{0}^{\infty} c_{i} N^{i}
$$

where $N^{i}$ is the product of $i$-copies of the number operator. With $\hbar=1$ $N$ is essentially the Hamiltonian of the quantum harmonic oscillator. This procedure quantises the classical Hamiltonian $h_{C} \in H_{C}$ and determines an appropriate operator ordering. The action of $H_{Q}$ on $L^{2}(x, d x)$ is well defined, 
the eigenfunctions of $H_{Q}$ are the familiar harmonic oscillator eigenfunctions, and the corresponding eigenvalues are simply $E_{n}$.

Although the coefficients of $H_{Q}$ in (2) depend on the choice of $h_{C} \in H_{C}$, only values of $h_{C}(z)$ when $z$ is a positive integer play a role in the dynamics. It does not matter which $h_{C} \in H_{C}$ we use to construct $H_{Q}$, the resulting quantum system will be the same. $H_{Q}$ is a unique quantum Hamiltonian, uniquely determined by the spectral sequence, and which we can identify with the equivalence class of classical Hamiltonians $H_{C}$.

This is a little surprising since it indicates that the correspondence of classical to quantum systems is not one-to-many as we might naievely have expected. The usual correspondence between classical observables $O_{C}(q, p)$, and quantum observables $O_{Q}(q, p, \hbar)$ is one-to-many in the sense that for a given classical Hamiltonian $h_{C}(q, p)$, there is an infinite number of corresponding quantum Hamiltonians $h_{Q}(q, p, \hbar)$ determined by the property $h_{Q}(q, p, 0)=h_{C}(q, p)$. In our construction the redundancy due to different choices of operator ordering does not arise. A different redundancy however does arise. This is because there are different classical systems $h_{C}$ which correspond to the single quantum system $H_{Q}$. This is directly attributable to the fact that many different continuous functions interpolate a function whose values are specified only on the integers. For a given value of Planc's constant the differences between these classical systems occur on a scale smaller than $\hbar$. For compact dynamical systems such as those used in our construction, the correspondence between classical and quantum systems is in fact many-to-many.

\section{Finite dimensional case}

It is straightforward to generalise the results of the previous section to the $k$-dimensional case. A natural generalisation of lemma 1 is given by

LEMMA 2 Given a $k$-indexed sequence of complex numbers $\left\{\zeta_{n_{1} \ldots n_{k}}: 0 \leq\right.$ $\left.n_{i} \in Z, 1 \leq i \leq k\right\}$ obeying a growth law $\left|\zeta_{n_{1} \ldots n_{k}}\right|<\exp \left(a+b_{1} n_{1}+\ldots+b_{k} n_{k}\right)$ for $a \in \Re, b_{i} \in \Re^{+}$, there exists an equivalence class $S$ of entire analytic functions on $C^{k}$ such that $s\left(n_{1}, \ldots, n_{k}\right)=\zeta_{n_{1} \ldots n_{k}}$ for all $s \in S$.

This leads to a natural generalisation of theorem 11 as follows. 
THEOREM 2 Given a $k$-indexed sequence of real numbers $\left\{E_{n_{1} \ldots n_{k}}: 0 \leq\right.$ $\left.n_{i} \in Z, 1 \leq i \leq k\right\}$, which obeys the growth law $\left|E_{n_{1} \ldots n_{k}}\right| \leq \exp \left(a+b_{1} n_{1}+\ldots+\right.$ $b_{k} n_{k}$ ) for some $a \in \Re, b_{i} \in \Re^{+}$, there exists an equivalence class of classically integrable $k$-dimensional non-linear oscillators $H_{C}$, where if $h_{i}^{0}$ for $1 \leq i \leq k$ are independent classical harmonic oscillator Hamiltonians, each $h_{C} \in H_{C}$ is of the form $h_{C}\left(h_{1}^{0}, \ldots, h_{k}^{0}\right)$ and its quantum spectral sequence is $E_{n_{1} \ldots n_{k}}$.

The proofs of lemma 2 and theorem 2 are based on a consideration of

$$
s\left(z_{1}, \ldots, z_{k}\right)=\sum_{n_{1} \ldots n_{k}=0}^{\infty} \zeta_{n_{1} \ldots n_{k}} f_{n_{1}}\left(z_{1}\right) \cdot \ldots \cdot f_{n_{k}}\left(z_{k}\right)
$$

where for $0<\epsilon_{i} \in \Re$ we have

$$
f_{n_{i}}\left(z_{i}\right)=\exp \left(\left(z_{i}-n_{i}\right)\left(2 \pi+b_{i}+\epsilon_{i}\right)\right) \frac{\sin \left(2 \pi\left(z_{i}-n_{i}\right)\right)}{2 \pi\left(z_{i}-n_{i}\right)},
$$

along with the usual technical provision at each of the removable singularities. If $S_{0}$ is the set of entire functions of $C^{k}$ which vanish at the points $\left(z_{1}, \ldots, z_{k}\right)=\left(n_{1}, \ldots, n_{k}\right)$ for $0 \leq n_{i} \in Z$ and $1 \leq i \leq k$, the equivalence classes have the form $S=s+S_{0}$. Once again they are independent of the explicit values of $a, b_{i}$ and $\epsilon_{i}$ used to construct them. The proofs proceed as before with only minor alterations and so we omit the explicit details.

\section{Berry's hypothesis}

Comparing the spectral rigidity of quantum systems which are classically integrable to those which are classically chaotic, Berry considered the spectrum of an unknown dynamical system whose energy levels are given by the imaginary parts of the zeros on the critical line of the Riemann zeta function [G]. It had previously been conjectured by Montgomery that the distribution of energy gaps of such a system would be GUE [7]. Montgomery's conjecture was supported numerically by the work of Odlyzko according to a report by Bohigas and Giannoni [8]. Since GUE statistics are normally associated with classically chaotic systems which do not possess time reversal invariance, this suggested to Berry that the corresponding unknown classical dynamical system must be chaotic [6]. Berry provided further support for this hypothesis through theoretical work based on a semi-classical consideration of the rigidity of its spectral sequence [6]. 
We will now show that although there may exist classically chaotic systems whose quantum spectrum is given by the imaginary parts of the nontrivial zeros of the Riemann zeta function, there also exists an infinite family of classical integrable systems for which this is true.

To see that this is so it suffices to show that the monotonic sequence $\zeta_{n}$ where $1 / 2+\imath \zeta_{n}$ is the $n$-th nontrivial zero of the Riemann zeta function satisfies the growth condition of theorem 1. This assertion follows immediately from a classical theorem about the distribution of zeros on the critical line due to Hardy and Littlewood [9]. Their theorem states that if $N(T)$ is the number of zeros of the Riemann zeta function on the interval $[1 / 2,1 / 2+\imath T]$, then there exists a constant $a$ so that

$$
N(T)>a T \text {. }
$$

This tells us that if $1 / 2+\imath \zeta_{n}$ is the position of the $n$-th zero, then

$$
\zeta_{n}<a^{-1} n
$$

The sequence $\left\{\zeta_{n}: 0 \leq n \in Z\right\}$ is therefore exponentially bounded and satisfies the requirements of theorem 1, which we apply to deduce the following.

THEOREM 3 There exists an infinite family of classically integrable nonlinear oscillators whose quantum spectrum is given by the imaginary part of the sequence of zeros on the critical line of the Riemann zeta function.

It is possible to go even further by relabelling the sequence with one index $\zeta_{n}$ as a sequence with $k$ indices $\zeta_{n_{1} \ldots n_{k}}$ for any $1 \leq k \in Z$, such that the growth condition of lemma 2 is still satisfied. There are many ways in which to do this and applying theorem 2 we deduce the following.

THEOREM 4 For any finite dimension $k$, there exists an infinite family of classically integrable $k$-dimensional non-linear oscillators whose quantum spectra reproduce the imaginary part of the zeros on the critical line of the Riemann zeta function.

\section{Conclusion}

Apart from an illustration of how the correspondence between classical and quantum dynamical systems is many-to-many rather than one-to-many, our 
main conclusion is that exceptions to the rule of Berry and Tabor regarding the distribution of energy gaps in the spectrum of a classically integrable system, are more numerous than the literature suggests. In particular we show that contrary to the Berry hypothesis, the unknown classical dynamical system whose chaotic quantum spectrum is given by the imaginary part of the non-trivial zeros of the Riemann zeta function is not unique and need not be chaotic. For a given value of Planc's constant and for any finite dimension of phase space, there exists an infinite number of classically integrable nonlinear oscillators whose quantum spectrum simulates that of Berry's unknown system. We conclude that the Poisson distribution of energy gaps is not a universal property of integrable systems, but of a restricted class of systems for which the approximations made by Berry and Tabor are valid. It would be of considerable interest to characterise more precisely the range of validity of their approximations and consequently of their result regarding the statistical distribution of quantum energy gaps of classically integrable systems.

\section{Acknowledgements}

This work was started while an EU-STF fellow at the Dept. of Mathematics in Kyoto University, and was finished at the Dept. of Mathematical Physics at University College Dublin. We would like to thank M. Jimbo and D. Judge for their hospitality, as well as T. Shiota, P. Mellon, J. Lewis, E. Buffet, B. Eckhardt and I. Guarneri for their comments and suggestions.

\section{References}

[1] B. Eckhardt: Quantum mechanics of classically non-integrable systems. Physics Reports. Vol. 163 No.4 June 1988.

[2] M.V. Berry \& M. Tabor: Proc. Roy. Soc. London, Ser A 356 p.357 1977.

[3] M. Razavy: Level spacing associated with a classically degenerate motion. Physics Letters Vol. 113A No. 6 p.297-298 Dec. 1985.

[4] G. Casati, B.V. Chirikov \& I. Guarneri: Energy level statistics of integrable quantum systems. Phys. Rev. Letters Vol. 54, No. 13 p.1350-1353. April 1985. 
[5] T. H. Seligman \& J. J. Verbaarschot: Phys. Rev. Letters 56, p.2767, 1986.

[6] M. V. Berry: Proc. R. Soc. London A 400 p.229-251 1985. Semicalssical theory of spectral rigidity.

Wiley \&

[7] H. L. Montgomery: Proceedings of Symposia in Pure Mathematics 24, p.181-193 1973.

[8] O. Bohigas \& M. J. Giannoni: Chaotic Motion and Random Matrices Lecture Notes in Physics Vol. 209 p.1-99 Springer Verlag.

[9] E. C. Titchmarsh: The theory of the Riemann Zeta Function. Oxford Clarendon Press 1951. Theorem 10.7. 